Check for updates

Cite this: Nanoscale Adv., 2019, 1, 3474

\title{
Fluorescence enhanced lab-on-a-chip patterned using a hybrid technique of femtosecond laser direct writing and anodized aluminum oxide porous nanostructuring $\dagger$
}

\author{
Zhi Yu, ${ }^{\text {ac }}$ Yuhao Lei, ${ }^{\text {a }}$ Weili Yu, ${ }^{\text {ac }}$ Jinluo Cheng, ${ }^{\text {ac }}$ Jun Xing, ${ }^{\text {ac }}$ Xin Zheng, ${ }^{\text {ac }}$ \\ Zhibing Zhan, ${ }^{\text {b }}$ Bin Wang ${ }^{\text {ac }}$ and Chunlei Guo (D) *abc
}

\begin{abstract}
In this paper, we demonstrate a simple yet effective hybrid method to fabricate lab-on-a-chip devices on aluminum (Al) foil. Instead of using conventional photoresists and lithography methods, an array of square units is first produced by femtosecond laser direct writing, followed by generating highly ordered anodized aluminum oxide (AAO) nanoporous structures within each unit. The AAO treated area becomes hydrophilic. Next, we functionalize the surrounding area outside the square units to superhydrophobic by electrochemical deposition and further chemical modification. This hydrophilic and hydrophobic pattern allows us to confine the liquid samples to be detected within the hydrophilic AAO detection area. We use rhodamine $6 \mathrm{G}(\mathrm{R} 6 \mathrm{G})$ as a probe, and obtain a fluorescence intensity enhancement from R6G by 70 times over a flat surface. This leads to the detection sensitivity of R6G molecules to a concentration as

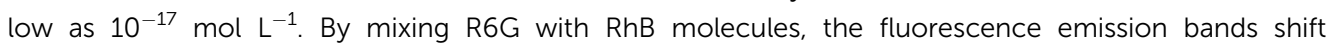
significantly due to the addition of RhB molecules, showing a significantly improved spectral resolution compared to traditional fluorescence spectrometers for liquid samples. This phenomenon can be attributed to the energy transfer between R6G and RhB under laser excitation, which was enhanced by the AAO nanostructures. The array-based LOC device demonstrated in this paper is simple and convenient to fabricate, has low sample consumption and dramatically enhances the fluorescence yield with improved spectral resolution.
\end{abstract}

Received 4th June 2019

Accepted 15th July 2019

DOI: $10.1039 / c 9 n a 00352 e$

rsc.li/nanoscale-advances

\section{Introduction}

The lab-on-a-chip (LOC) system has attracted tremendous attention in various research fields, such as biology, chemistry, medical science $e t c .{ }^{1-3} \mathrm{~A}$ LOC system, composed of array-based, continuous microfluidics, encoded bead and digital microfluidics platforms, can detect, integrate, separate, and process biological and chemical samples in order to achieve more complex analyses. ${ }^{4-7}$ Each LOC device can be assembled with other types of platforms for more efficient sample handling and analysis. A LOC usually includes many repetitive structure units for high-throughput analysis with a small volume of samples, and the advantages of these miniaturized analytical systems include accuracy improvement, multiplexing, lower sample and

${ }^{a}$ The Guo China-US Photonics Laboratory, Changchun Institute of Optics, Fine Mechanics and Physics, Chinese Academy of Sciences, Changchun, China, 130033

${ }^{b}$ The Institute of Optics, University of Rochester, Rochester, NY 14627, USA. E-mail: guo@optics.rochester.edu

${ }^{c}$ State Key Laboratory for Applied Optics, Changchun Institute of Optics, Fine Mechanics and Physics, Chinese Academy of Sciences, Changchun, China, 130033

$\dagger$ Electronic supplementary information (ESI) available. See DOI: 10.1039/c9na00352e reagent consumption, disposability and decreased analysis times. ${ }^{8-10}$ Array-based LOC detection systems are mostly functionalized by further integration procedures, and exhibit special physical and chemical properties. Such integrated devices can realize the detection and analysis in some specific fields with much better sensitivity and resolutions. In the past, larger scale structures were typically prepared with lithography methods, which require masks and multi-step processes. ${ }^{11-13}$ It can be rather complicated when frequent patch size adjustments are needed, thus leading to several disadvantages, such as nonreusability and adsorption of organic compounds. ${ }^{14}$ On the other hand, femtosecond laser direct writing is a clean method to produce a high-quality cut with arbitrary shape and dimension. ${ }^{15}$

During the past few decades, we have witnessed tremendous developments of ultrafast lasers, which have become more userfriendly and cost effective. ${ }^{16}$ Ultrafast femtosecond lasers have occupied a very important position in materials machining. Although nanosecond lasers were commonly used in machining, the thermal effects from machining with relatively long ns-lasers can lead to a larger heat-affected zone, lower machining quality, and greater collateral damage such as 
stresses, cracks, and molten layer recast and slag. ${ }^{17}$ By contrast, femtosecond lasers provide an ideal machining quality with high spatial resolution and minimal thermal damage. Due to the ultrafast pulse length, femtosecond lasers can deliver energy in a controllable manner. ${ }^{18}$ The heat diffusion around the machined area is minimal and the heat-affected zone is very small with a small amount of molten material and a low level of thermal defects left behind. ${ }^{19}$ They show much better advantages than traditional laser processing techniques, specifically, energy deposition in a time scale shorter than that for electronphonon coupling processes in any material due to the ultrashort pulse width which can suppress the formation of the heataffected zone allowing laser processing with high precision and resolution. ${ }^{20}$ The unique advantage of femtosecond laser processing over conventional methods resides in the capability of sculpting complex three dimensional shapes at micro- and nanoscales in transparent materials, both for inorganic and organic materials. ${ }^{\mathbf{2 1 2 2}}$ One can precisely set the interaction region in a localized area of either surfaces or in volume by employing focused ultrashort pulses with extremely high peak intensities for spot machining. ${ }^{15,23,24}$ As a consequence, femtosecond laser technologies have been increasingly developed and extensively applied to the fabrication of LOC devices and integration of multifunctional components or specific functional materials into localized positions of LOC devices with a high degree of precision and reproducibility. ${ }^{25}$ The femtosecond laser processing technology has been widely used in the fabrication or integration of many kinds of LOC devices, such as SERS, optofluidic, electrochemical and biological function detection chips. ${ }^{26-29}$

Fluorescence-based LOC detection chips have many practical applications by using fluorescence-based detection techniques including fluorescence resonance energy transfer, fluorescence quenching, fluorescence polarization, timeresolved fluorescence and fluorescence fluctuation spectroscopy. ${ }^{30-34}$ As for a single component sample, quantitative analysis or the change of the molecular structure could be easily realized just by the intensity or band shift of the fluorescence spectra. Therefore, under these conditions, researchers mostly focus on the study of increasing the detection sensitivity of fluorescence analyses through the integration of materials possessing fluorescence enhancing properties on the chip. However, for the analysis of mixed components, not only the detection sensitivity but also the distinguishability is of great importance, especially when the emission band position of the mixed samples is located too close to be distinguished. Porous anodized aluminum oxide (AAO), fabricated by anodizing an $\mathrm{Al}$ substrate in an appropriate acid solution with the formation of a self-ordered nano-channel structure, has been reported to exhibit fluorescence enhancement properties when fluorophores are in proximity to its surface..$^{35-37}$ Different from metal-enhanced fluorescence, which requires a spacer between the metal surface and fluorophores to avoid the quenching effect, the AAO surface offers the maximum fluorescence enhancement when the fluorophores are directly in contact with it. Based on previous studies, the enhancement mechanism is probably attributed to the following main reasons: (1) the optical scattering effect of the AAO surface, which causes the redistribution of electromagnetic fields with high surface intensities, may play a very important role in the fluorescence enhancement; ${ }^{38-40}$ (2) the fluorescence enhancement might result from the evanescent electric field from the surface of the AAO nanoporous structures, observed in metal oxide nanoscale materials. ${ }^{41-44}$ Upon integrating AAO nanoporous thin films into LOC devices, the detection efficiency and functionality will be greatly increased due to the fluorescence enhancement. However, such an integration is difficult.

Here, we combine femtosecond laser direct writing and AAO fabrication techniques to develop a rapid and high-precision multi-scale fabrication technique, allowing us to obtain a LOC device with significant signal enhancement and spectral resolution. In this work, we use the femtosecond laser direct writing technique to fabricate an AAO array-based LOC device, which includes a $162 \mathrm{~mm} \times 2 \mathrm{~mm}$ AAO detection platform arranged into a $4 \times 4$ array. By choosing R6G as a fluorescence probe molecule, the fluorescence intensity of the molecule based on the as-synthesized structure is 70 times stronger than that of a flat surface. Furthermore, this platform also exhibits much

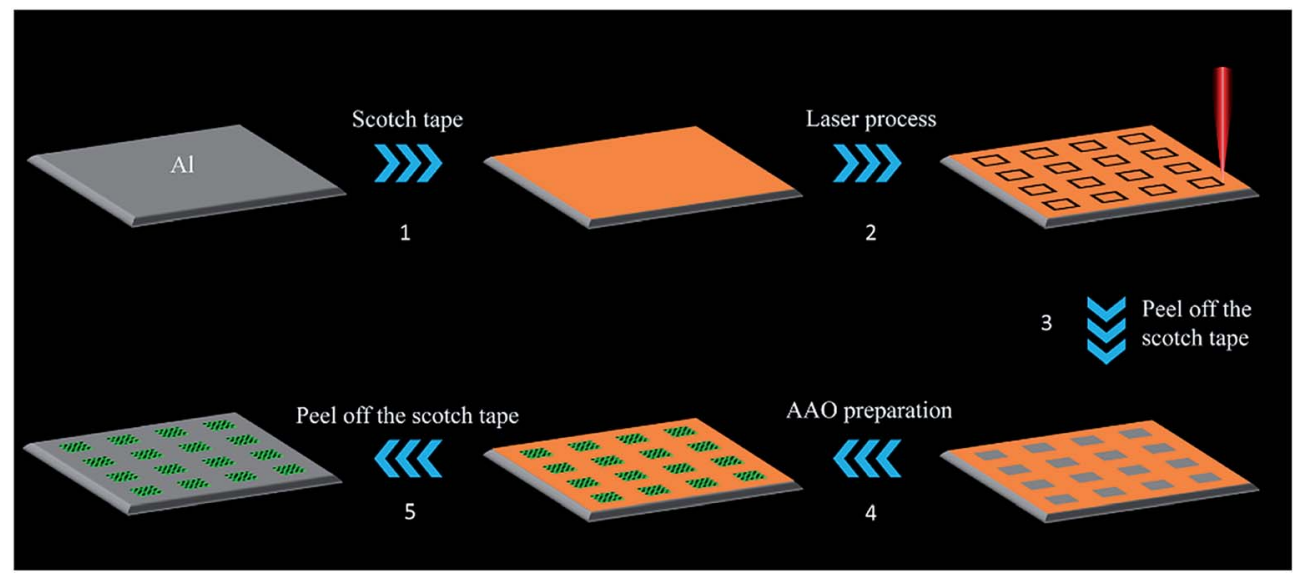

Fig. 1 Schematic for the fabrication of the AAO array-based LOC device. 
better resolution in distinguishing mixed components (R6G and $\mathrm{RhB}$ ) than traditional spectrometric analyses in liquid samples. Thus, besides a simple and efficient method proposed for fabrication, the as-synthesized AAO array-based LOC detection device exhibits strong fluorescence enhancement and provides a new method for analyzing mixed components.

\section{Experimental section}

\section{Materials}

Al foil $(0.2 \mathrm{~mm})$ was purchased from Zhonguo New Material (Beijing) Technology Co. Ltd. Perchloric acid $\left(\mathrm{HClO}_{4}\right)$, phosphoric acid, chromium hemitrioxide $\left(\mathrm{Cr}_{2} \mathrm{O}_{3}\right)$, oxalic acid, silver nitrate $\left(\mathrm{AgNO}_{3}\right)$, rhodamine $\mathrm{B}(\mathrm{RhB})$, rhodamine 6G (R6G), $1 H^{\prime}, 1 H^{\prime}, 2 H^{\prime}, 2 H^{\prime}$-perfluorodecanethiol (97\%) and ethyl alcohol (98\%) were purchased from Sigma-Aldrich Co. Ltd.

\section{Preparation of the AAO array detection chip}

The fabrication of the AAO array detection chip is shown in Fig. 1. We first cut $\mathrm{Al}$ foil into an $18 \times 18 \mathrm{~mm}$ square, and attached Scotch tape on the surface. Then we use a femtosecond laser processing system, as shown in Scheme $\mathrm{S} 1, \dagger$ to directly write the borders of square units which are arranged into a $4 \times$ 4 grid array on the Scotch tape surface. Afterwards, we peel off the Scotch tape inside the squares to expose the $\mathrm{Al}$ foil. Before anodization, the substrate was electropolished in a $1: 7$ volume mixture of $\mathrm{HClO}_{4}$ and $\mathrm{C}_{2} \mathrm{H}_{5} \mathrm{OH}$. After that, porous alumina membranes were prepared in a two-step anodizing process: the substrate after polishing was immersed in a $0.3 \mathrm{~mol} \mathrm{~L}^{-1}$ oxalic acid electrolyte with $\mathrm{Pb}$ as a cathode. The anodizing voltage was maintained at $40 \mathrm{~V} \mathrm{DC}$ at ambient temperature; after $6 \mathrm{~h}$ of anodization, we immerse the substrate in a mixture of $6 \%$ phosphoric acid and $1.8 \%$ chromic acid at $60{ }^{\circ} \mathrm{C}$ for $10 \mathrm{~min}$ to remove the aluminum oxide layer, and repeat the first step. Finally, we peel off the remaining Scotch tape to obtain the AAO array detection chip.

\section{Surface wettability treatment}

The as-prepared AAO detection chip was then immersed in a silver nitrate electrolyte with $\mathrm{Al}$ as the cathode under at $1 \mathrm{~V} \mathrm{DC}$ voltage at ambient temperature (Fig. 2). Electrochemical deposition took place only at the $\mathrm{Al}$ foil area outside the AAO regions. After 10 min of electrochemical reaction, the chip was washed with deionized water and dried under a $\mathrm{N}_{2}$ flow. Finally, the chip was immersed in $1^{\prime} H, 1^{\prime} H, 2^{\prime} H, 2^{\prime} H$-perfluorodecanethiol solution $\left(10^{-3} \mathrm{M}\right.$ in ethanol) for $6 \mathrm{~h}$ followed by washing and drying steps.

\section{Characterization of the fluorescence enhancement properties of the AAO chip}

The fluorescence enhancement properties are characterized by using probe molecules R6G at a concentration of $10^{-6} \mathrm{~mol} \mathrm{~L}^{-1}$. Each measurement uses $2 \mu \mathrm{L}$ solution, dropped on both the square area of the AAO chip and Al substrate with the same square area as a reference. Fluorescence spectra of the samples were collected after air drying.
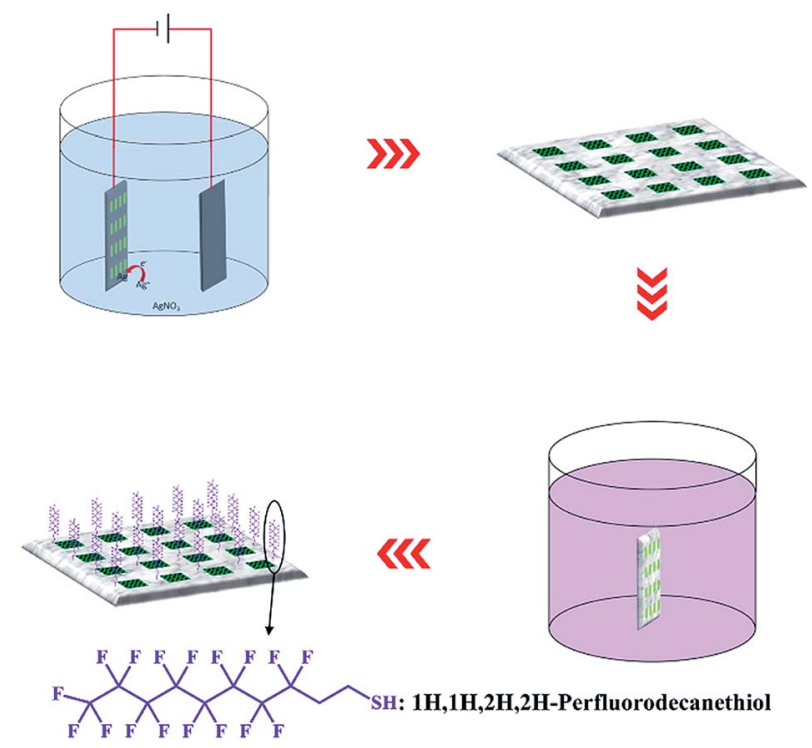

Fig. 2 Schematic of the surface wettability treatment process.

Concentration dependent fluorescence spectra of R6G molecules based on the AAO chip

The fluorescence spectra were measured for diluted R6G solutions with concentrations of $10^{-11}, 10^{-12}, 10^{-13}, 10^{-14}, 10^{-15}$, $10^{-16}, 10^{-17}$ and $0 \mathrm{~mol} \mathrm{~L}^{-1}$; for the characterization, the data were taken for air dried samples with $2 \mu \mathrm{L}$ solution. The UV-vis and fluorescence spectra of the R6G liquid sample with different concentrations were collected at the same time.

\section{Characterization and measurements}

A commercial Ti:sapphire laser system with chirped-pulseamplification (Spitfire Ace, Spectra Physics, Santa Clara, CA, USA) is employed as a light source for direct laser writing, which delivers horizontally polarized femtosecond laser pulses with a repetition rate of $1 \mathrm{kHz}$, a central wavelength at $800 \mathrm{~nm}$, and a time duration of $40 \mathrm{fs}$. The maximum energy of each laser pulse is $7 \mathrm{~mJ}$. Neutral density filters and a half-wave plate were used to control the pulse energy and the direction of the laser polarization, respectively. The laser beam was focused using an objective lens (plan fluorite objective, $4 \times$, N.A $=0.1, f=17.2$ $\mathrm{mm}$, Nikon, Tokyo, Japan) at normal incidence.

Confocal fluorescence spectroscopy and mapping analyses were performed with a LabRAM HR Evolution Raman spectrometer (HORIBA Jobin/Yvon) in fluorescence mode with a radiation of $532 \mathrm{~nm}$. The signals were detected with $180^{\circ}$ geometry using a multichannel air-cooled $\left(-70{ }^{\circ} \mathrm{C}\right)$ chargecoupled device (CCD) camera. UV-vis spectra of the liquid sample were collected using a Cary 5000 UV-vis-NIR spectrometer (Agilent, USA). Fluorescence spectra of the liquid sample were collected using a Cary Eclipse Bundle (Agilent, USA) with a long life Xe flash lamp and an extended wavelength range PMT (high sensitivity R928) provided as standard. Scanning electron microscope (SEM) characterization was carried out by using an S48000 (Hitachi, Japan), and atomic force microscopy (AFM) characterization was carried out on a MULTIMOD E8 (Bruker, 
USA). The contact angle (CA) was measured using a POWEREACH JC2000D3 contact angle measurement device. The fluorescence quantum yield was measured using an FL920 fluorescence spectrometer from $528 \mathrm{~nm}$ to $700 \mathrm{~nm}$. A Xe lamp was utilized as the excitation light source with a fixed wavelength at $532 \mathrm{~nm}$.

\section{Finite difference time domain method simulations}

For the simulations, we have employed the finite-differencetime-domain (FDTD) method based on Yee's algorithms. In the FDTD method, Maxwell's equations are discretized in both time and space. A commercial FDTD package known as Lumerical Solutions (Lumerical Inc.) was used to calculate the electric field distribution of AAO. Perfectly matched layers were used to absorb the scattered radiation in the $Z$-direction, and periodic boundary conditions were used to perform periodic AAO arrays in $X$ and $Y$ directions. The models are illuminated with a $\mathrm{p}$ polarized plane wave propagating along the $Z$ axis and polarizing along the $X$ axis from the top of AAO. A detector box was placed on the surface to measure the distribution $\left(\left|E_{\mathrm{x}}\right|\right)$ of the electric field. The source of dipoles was placed on the AAO surface which has different intensities of electric field to analyse the interaction between the molecules and AAO substrate. Ultrafine mesh sizes $(\Delta x=1 \mathrm{~nm}, \Delta y=1 \mathrm{~nm}, \Delta z=10 \mathrm{~nm})$ were used in the simulations. A refractive index of $n=1.0$ is chosen for air to generate an asymmetric environment. The optical constants of $\mathrm{Al}$ and $\mathrm{Al}_{2} \mathrm{O}_{3}$ were taken from Palik (Handbook of Optical Constants of Solids).

\section{Results and discussion}

\section{Surface morphology characterization of the AAO detection chip}

The prepared samples are characterized by optical imaging, SEM, and AFM surface morphology analysis, as shown in Fig. 3. As the preparation procedure shown in Fig. 1, after step 2 and step 3, the Scotch tape of the square area was peeled off leaving the $\mathrm{Al}$ foil exposed. As a result, during the following polishing and anodization process, remnants of the Scotch tape can prevent the $\mathrm{Al}$ foil under the Scotch tape from touching the reagent and all the reactions take place only in the square area. Thus, after polishing and anodization procedures (step 4, Fig. 1), a $2 \mathrm{~mm} \times 2 \mathrm{~mm}$ square area AAO array chip was successfully prepared on $\mathrm{Al}$ foil spaced $2 \mathrm{~mm}$ apart (Fig. 3A).

Fig. 3B shows the AFM morphology of the AAO nanostructure, which indicates a very uniform nanoporous structure. Fig. 3C and D show the SEM image of the surface and cross section of the AAO nanotemplate. It can be seen from this image that the AAO substrate exhibits a perfectly porous array structure with an average diameter of about $50 \mathrm{~nm}$ for each pore. Fig. 3D indicates that the depth of the AAO nanostructure is $44.8 \mu \mathrm{m}$. The inset in Fig. 3D shows the amplified figure of the cross section, which is to better observe the structure. It should be mentioned that the depth of the AAO nanopores varied with the oxidation time; the oxidation time used here is $4 \mathrm{~h}$. In addition, the AAO nanoporous template is grown on the base of $\mathrm{Al}$ foil, so the nanopores on the upper surface are open, while those on the bottom surface are closed.
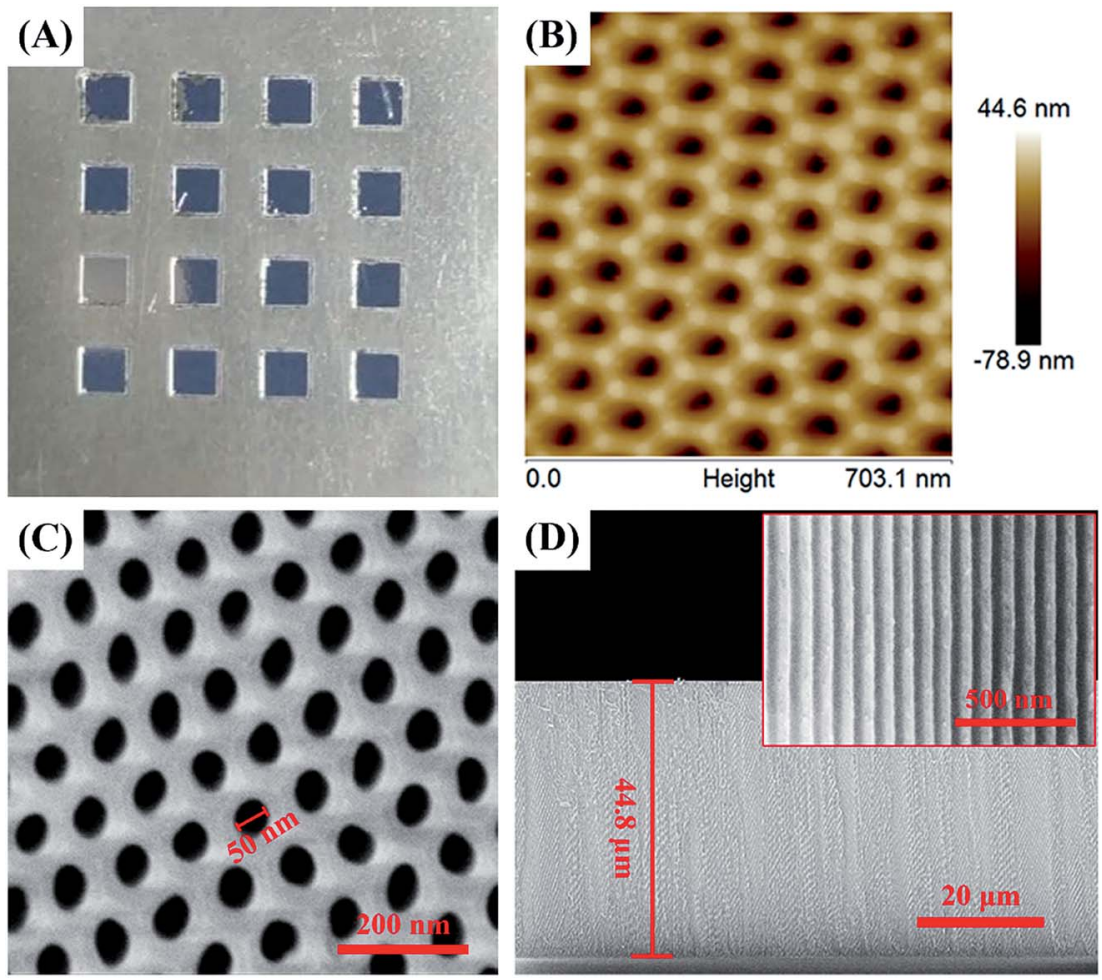

Fig. 3 Characterization of the AAO detection array chip: (A) optical image, (B) AFM image, (C) SEM image and (D) the section SEM image of the AAO nanostructures. 


\section{Surface wettability treatment and characterization}

To increase the detection accuracy and repeatability of this array chip, the Al surface surrounding the AAO units is further hydrophobically treated. This process can ensure that all liquid samples are limited among the AAO units during detection analyses. As shown in Fig. 2, hydrophobic treatment is performed by electrochemically depositing rough $\mathrm{Ag}$ nanostructures on the Al foil surface. Due to the high resistance, this procedure does not affect the AAO regions. Electrochemical deposition took place only on the Al foil part and the Ag nanostructure grew in this area. As a result, the surface of $\mathrm{Al}$ was covered with a $\mathrm{Ag}$ layer. The surface of $\mathrm{Al}$ foil is further characterized by SEM and EDS analyses, as shown in Fig. S1; $\dagger$ the surface is covered with rough Ag nanostructures. Then after immersing the substrate in the $1^{\prime} H, 1^{\prime} H, 2^{\prime} H, 2^{\prime} H$-perfluorodecanethiol solution, the sulfhydryl group of this molecule can combine with $\mathrm{Ag}$ due to the strong covalent bond between $-\mathrm{SH}$ and $\mathrm{Ag}$. This reaction can lead to the modification of $1^{\prime} H, 1^{\prime} H, 2^{\prime} H, 2^{\prime} H$-perfluorodecanethiol on the surface of the $\mathrm{Al}$ foil, and decrease the surface energy. Furthermore, the increase of surface roughness can also affect the surface wettability. With such a treatment, the Al foil surface becomes superhydrophobic.

The wettability of such a surface is then characterized by a contact angle measurement. Before the measurement, a $2 \mu \mathrm{L}$ water droplet hanging over the tip of the injector is used to test the wettability by moving towards the $\mathrm{Al}$ foil surface and touching and then moving away (Fig. 4A-E). The red arrows show the movement direction. During this process, the droplet is repelled by the $\mathrm{Al}$ surface. Thus, the test shows that the hydrophobic treatment gives the surface of the Al foil possesses superhydrophobic properties with extremely low adhesiveness.

As for the AAO square part, the contact angle measurement result shows a $\mathrm{CA}=83^{\circ}$ (Fig. $4 \mathrm{~F}$ ), which indicates that $\mathrm{AAO}$ has hydrophilic properties with much better adhesiveness than the hydrophobically treated $\mathrm{Al}$ foil part. Fig. 4G shows the optical image for the $10^{-3}$ mol L ${ }^{-1} \mathrm{RhB}$ solution dissolved in water standing on the AAO units even when the chip is tilted.
Therefore, the surface wettability treatment can limit the detection liquid sample to the AAO square area, due to the repellency of its surrounding superhydrophobic $\mathrm{Al}$ foil.

\section{Enhanced fluorescence phenomenon on the AAO nanoarray structure}

The effect of the AAO nanoarray structure on the fluorescence properties is studied for the R6G molecule $\left(10^{-6} \mathrm{~mol} \mathrm{~L}^{-1}\right.$, in water) as a probe molecule. Fig. 5 shows the molecular structure, absorption and fluorescence spectra of R6G molecules.

$2 \mu \mathrm{L}$ solution was dropped on the surface of the AAO square area which is the same amount of molecules dropped on the $\mathrm{Al}$ foil with the same area. Confocal fluorescence spectra were then collected. Fig. 6 shows the fluorescence spectra of R6G molecules adsorbed on the AAO substrate and $\mathrm{Al}$ foil, under the same conditions. A huge enhancement (70 times by the intensity) is observed and this indicates that AAO with porous nanostructures can enhance the fluorescence intensity of the adsorbed molecules. Fluorescence mapping analyses are also carried out for the R6G molecule adsorbed on the AAO and Al surface in a $30 \times 30 \mu \mathrm{m}$ area, and the results are shown in Fig. 6B and C, separately. The chroma of the mapping result in Fig. $6 \mathrm{~B}$ is much stronger than that in Fig. 6C, and shows a very uniform color distribution. It indicates that the AAO surface shows very homogeneously enhanced fluorescence properties, which ensure a much better detection accuracy of the AAO substrate.

\section{Effect of the anodization time on the fluorescence enhancement}

By controlling the anodization time, the parameters of the porous nanostructures in the AAO substrate can be effectively changed. We try to understand how these parameters affect the fluorescence and to find the best fluorescence enhancement conditions. In the fabrication of the AAO detection chip, we used various second anodization times of 1, 2, 3 and 4 h. From

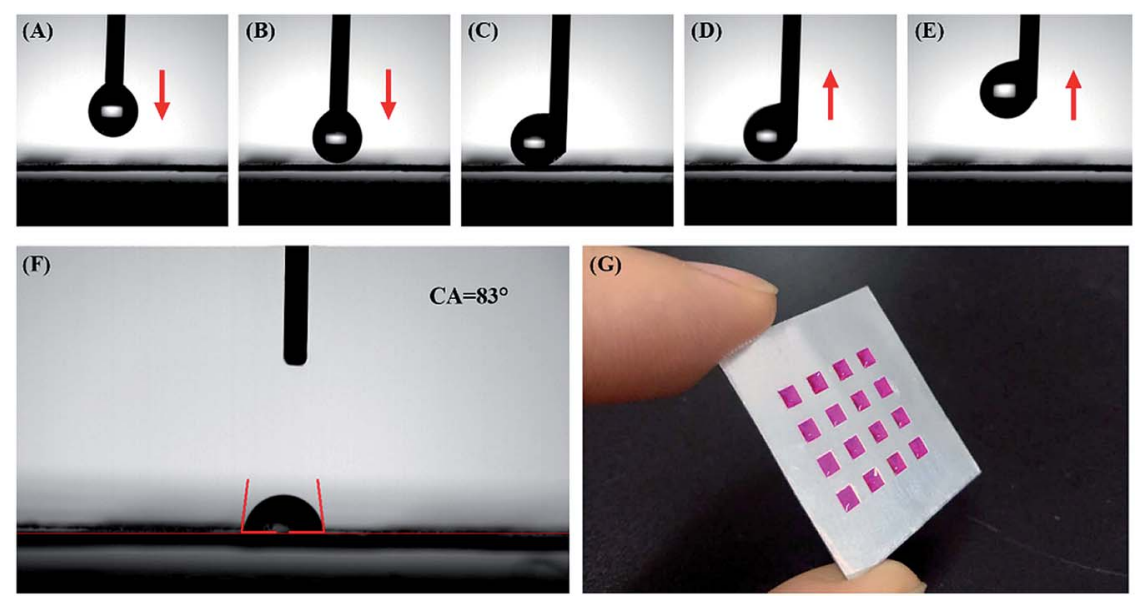

Fig. 4 Surface wettability characterization of the Al foil surface after hydrophobic treatment; the arrows label the injector movement direction $(A-E)$, the square $A A O$ substrate $(F)$ and the optical image of the $A A O$ array detection chip with a droplet of $10^{-3}$ mol $L^{-1} \mathrm{RhB} \mathrm{solution}$ dissolved in water supported on each detection AAO unit (G). 
(A)

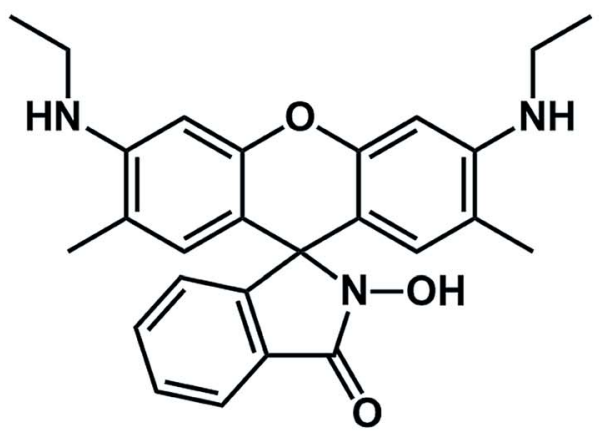

Rhodamine 6G
(B)

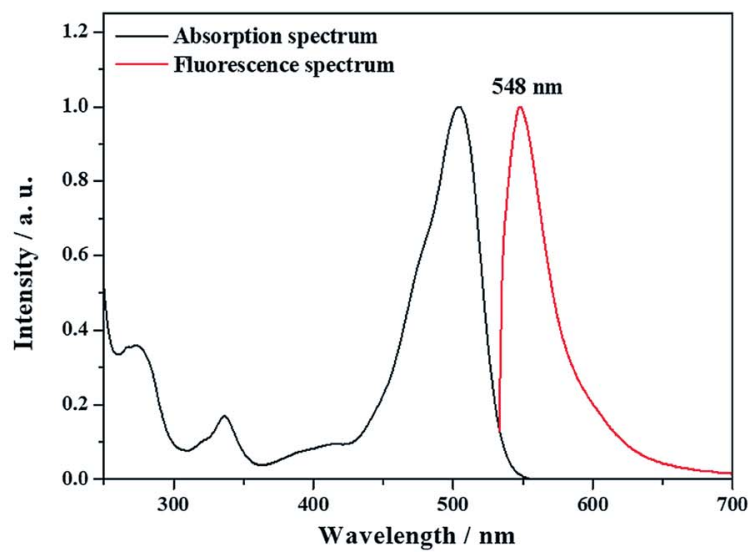

Fig. 5 Molecule structure (A) and absorption and fluorescence spectra (B) of R6G molecules.

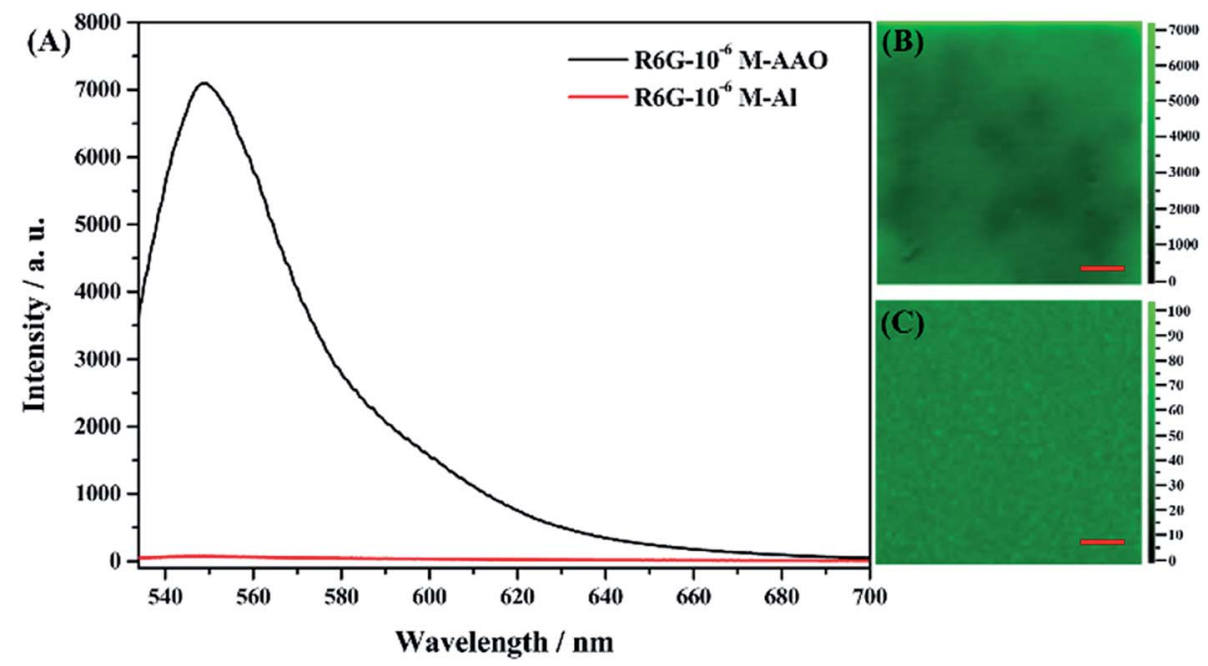

Fig. 6 (A) Fluorescence spectra of the molecule adsorbed on the AAO and Al surface. (B) Fluorescence mapping for AAO and (C) Al foil. The scale bar indicates $30 \mu \mathrm{m}$.

the SEM image of the surface and intersecting surface of the AAO substrate (Fig. S2 $\dagger$ ), the four substrate samples exhibit nearly the same porous structure with $50 \mathrm{~nm}$ diameter, but the thickness of the AAO substrate increases with increasing anodization time.

The fluorescence spectra of these four samples are recorded and the results are shown in Fig. 7A. The fluorescence changes with the anodization time, and the substrate prepared with $3 \mathrm{~h}$ of anodization shows the strongest enhancement. However, it is also shown from the mapping analyses (in Fig. 7B) that the AAO substrate after $4 \mathrm{~h}$ of anodization exhibits the best uniformity. Because the uniformity is much important for achieving a better detection accuracy, we choose $4 \mathrm{~h}$ of anodization time as the best substrate preparation conditions for our following studies, though the fluorescence intensity is not the strongest one.

\section{The enhancement mechanism of the AAO nanoporous} structures

As previously reported, the enhancement mechanism of the AAO nanostructures was not clearly described. Some attributed the mechanism to the following two reasons: (1) the optical scattering effect of the AAO surface, which causes the redistribution of the electromagnetic fields with high surface intensities, may play a very important role in the fluorescence enhancement; ${ }^{38-40}$ (2) the fluorescence enhancement might result from the evanescent electrical field or local electromagnetic field enhancement from the surface of the AAO nanoporous structures, observed in metal oxide nanoscale materials. ${ }^{4-44}$ We believe that both these mechanisms play important roles in the fluorescence enhancement. Thus, to understand the enhancement mechanism, the electromagnetic field distribution of the AAO nanoporous structures was stimulated by the FDTD method, as shown in Fig. 8 .

Here, the diameter of the nanopores is $50 \mathrm{~nm}$ while the thickness of the template is $44.8 \mu \mathrm{m}$ (measured from SEM images). The local electromagnetic field enhancement is defined as $|E|^{2} /\left|E_{0}\right|^{2}$ and it can be calculated from the results that the maximum enhancement of the AAO nanopores is about 68 fold that of the Al flat surface. This result is close to the experimental results we obtained. Thus, the enhancement 


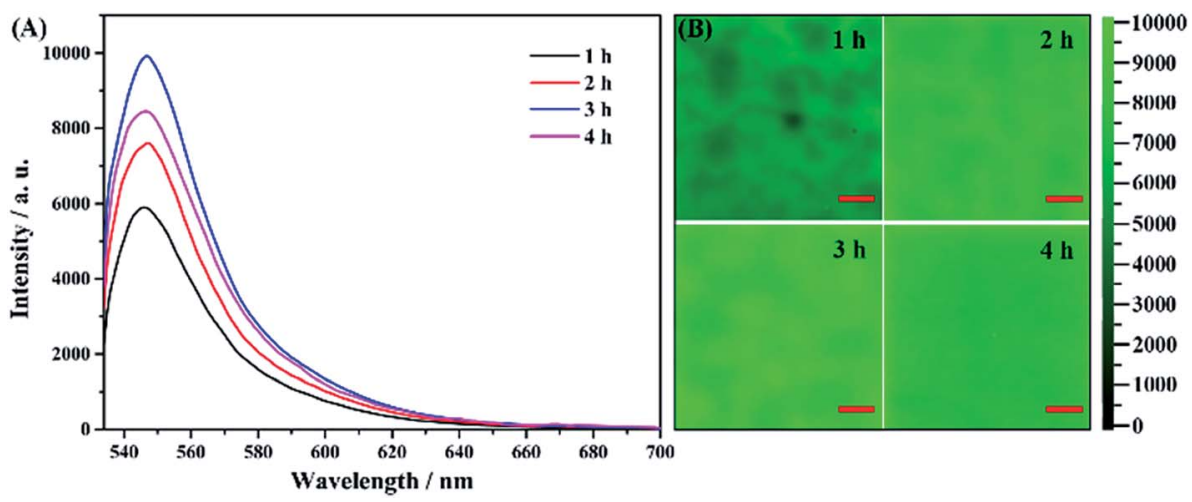

Fig. 7 The fluorescence intensity of R6G molecule adsorption on the AAO chip with different anodization times (1, 2, 3 and 4 h) (A) and the mapping analyses of each sample (B). The scale bar indicates $30 \mu \mathrm{m}$.

(A)

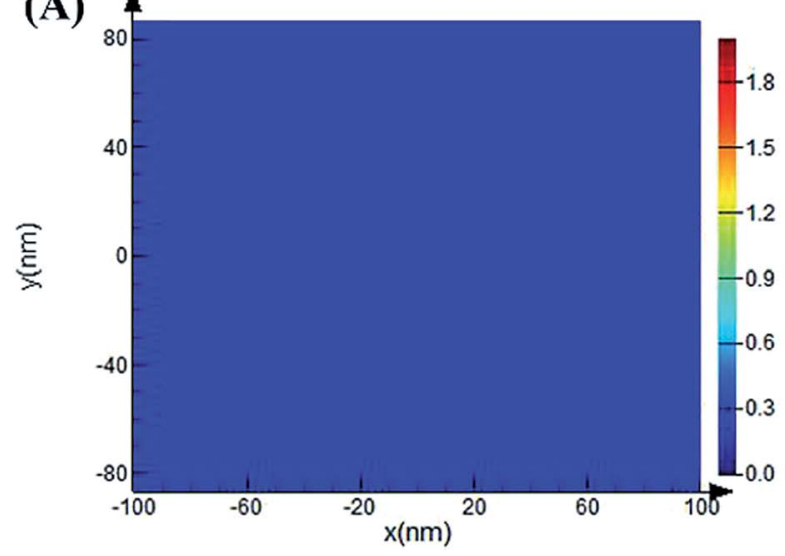

(B)

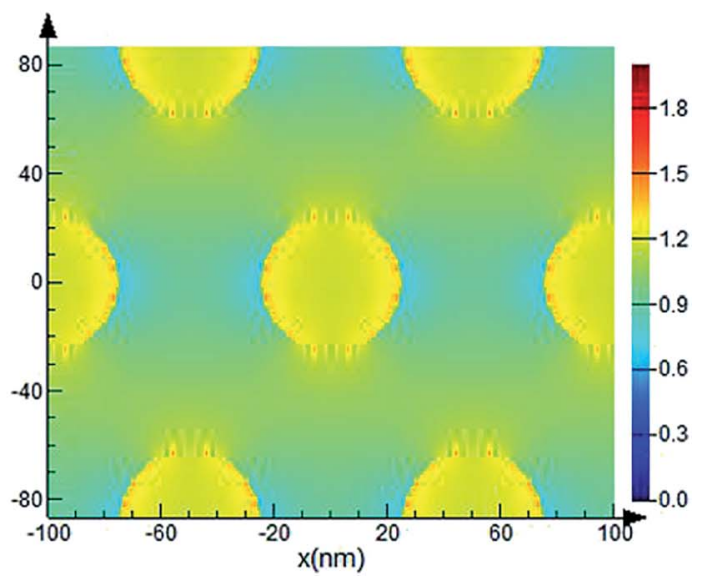

Fig. 8 Simulated electromagnetic field distribution of the flat Al surface (A) and AAO nanoporous structures (B) (50 nm, $4 \mathrm{~h}$ ) by the FDTD method. The incident light wavelength is $532 \mathrm{~nm}$.

mechanism can be attributed to the local electromagnetic field of the AAO nanoporous structures. The electromagnetic field distribution of R6G molecules, at its maximum emission of 548 $\mathrm{nm}$, adsorbed on the AAO surface at different positions compared to that on the Al surface was further investigated. As Fig. S3 $\uparrow$ shows, the intensity of R6G molecules on the AAO surface is stronger than that on the flat $\mathrm{Al}$ surface. And the intensity distribution is coincident with the electromagnetic field distribution of the AAO nanostructures under the excitation of a $532 \mathrm{~nm}$ laser. As calculated from Fig. $\mathrm{S} 3, \uparrow$ the intensity of R6G molecules is 1.1 times stronger than that on the $\mathrm{Al}$ surface. This indicates that the AAO nanostructures show no obvious enhancement in the emission of the R6G molecules without the $532 \mathrm{~nm}$ excitation laser. Thus, it can be concluded that the fluorescence enhancement mainly arises from the electromagnetic field excited by a $532 \mathrm{~nm}$ laser. The simulation of the electromagnetic field distribution of the AAO nanoplate with different thicknesses was further proposed. The results are shown in Fig. 9. As the figure shows, the intensity change of the electromagnetic field with different thicknesses coincides with the experiments shown in Fig. 7.

To further evaluate the fluorescence enhancement properties, the fluorescence quantum yield (QY) of the $\mathrm{Al}_{2} \mathrm{O}_{3}+\mathrm{R} 6 \mathrm{G}$ system was obtained. As Fig. S4 $\uparrow$ shows, the QY of R6G molecules adsorbed on the $\mathrm{Al}_{2} \mathrm{O}_{3}$ nanostructures is $18.17 \%$ while those on the $\mathrm{Al}$ surface hardly show obvious quantum yield results. This is because the light source used in the measurement is a Xe lamp and the photon density is not as strong as the $532 \mathrm{~nm}$ laser used in our experiment. Thus, the fluorescence quantum yield of R6G molecules on Al cannot be detected. However, $18.17 \%$ QY of R6G molecules adsorbed on $\mathrm{Al}_{2} \mathrm{O}_{3}$ indicates that the fluorescence properties of $\mathrm{Al}_{2} \mathrm{O}_{3}$ nanostructures can further enhance the $\mathrm{QY}$ of the adsorbed molecules.

\section{Concentration dependent enhanced fluorescence analyses}

Here we study the detection sensitivity for the fluorescence of the AAO substrate, by varying the R6G molecule concentrations to $10^{-11}, 10^{-12}, 10^{-13}, 10^{-14}, 10^{-15}, 10^{-16}, 10^{-17}$ and $0 \mathrm{~mol} \mathrm{~L}^{-1}$. The results are shown in Fig. 10. As the figure shows, the fluorescence intensity of the R6G molecules adsorbed on the AAO surface increased with increasing concentration of the molecules. The intensity changes logarithmically depending on the concentration, as shown in Fig. 10B, where the fluorescence intensity at 547 $\mathrm{nm}$ is plotted as a function of concentration, with a fitting relation $Y=-103 \log (X)+1776$. Obviously, the fluorescence intensity 
(A)

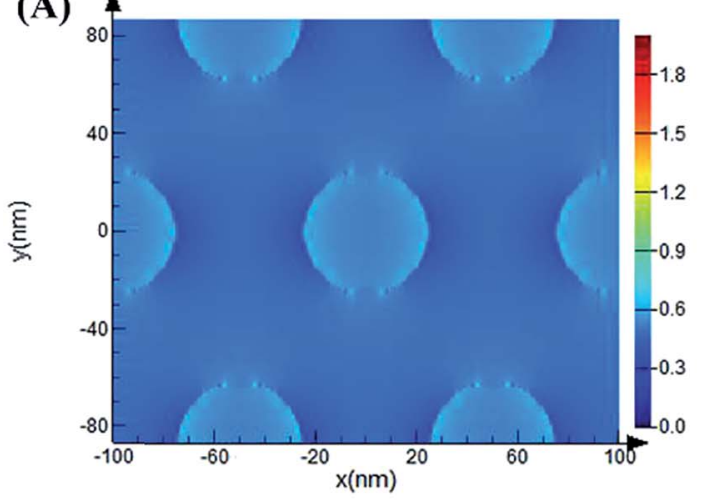

(C)

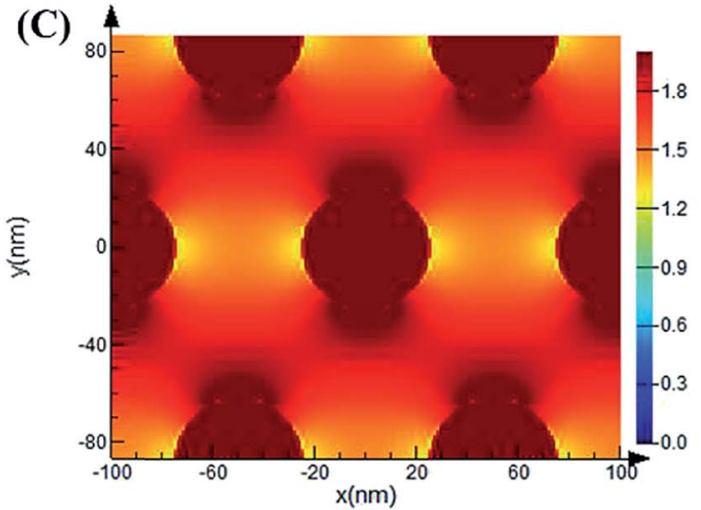

(B)

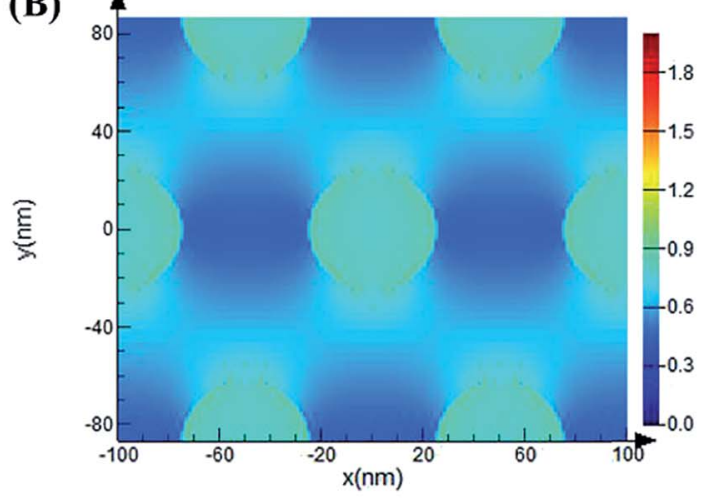

(D)

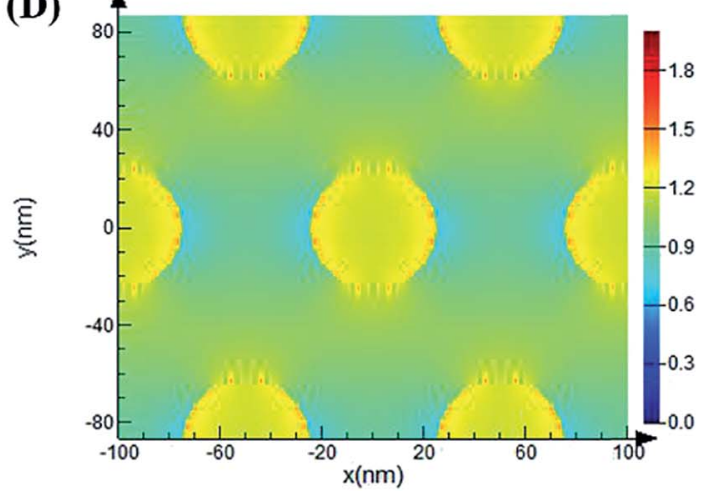

Fig. 9 Simulated electromagnetic field distribution of AAO nanopores with different thicknesses: (A) $11.2 \mu \mathrm{m}$, (B) $22.4 \mu \mathrm{m},(\mathrm{C}) 33.6 \mu \mathrm{m}$, and (D) $44.8 \mu \mathrm{m}$, by the FDTD method. The incident light wavelength is $532 \mathrm{~nm}$.

is detectable even when the R6G molecule concentration is $10^{-17}$ mol L ${ }^{-1}$. The sensitivity of the as-synthesized AAO structure can be clearly seen by comparing it with the detection using UV-vis spectra and fluorescence spectra of R6G molecules of the liquid sample collected using a common spectrometer. The UV-vis analysis results of the latter method are shown in Fig. S5, $\uparrow$ and the signal is almost not observable when the concentration of R6G molecules decreased to $10^{-10} \mathrm{~mol} \mathrm{~L}^{-1}$.

Meanwhile, the concentration dependent fluorescence spectra of R6G shown in Fig. S6† measured by the fluorescence spectrometer for liquid sample exhibits a $10^{-9} \mathrm{M}$ detection limit for R6G molecules. It can be thus concluded that the AAO porous nanostructure substrate exhibits obviously enhanced fluorescence properties. Fig. 10C shows the mapping analysis results for the fluorescence detection of R6G molecules adsorbed on each unit. As the figure shows, with the concentration of R6G molecules decreasing, the image color faded, which revealed that the fluorescence intensity of the R6G molecules in the AAO units decreased. Meanwhile, the color of the mapping analysis image is quite uniform for each concentration. This result can further prove that this method is suitable for fluorescence imaging analysis.

\section{Fluorescence detection of the mixture of R6G and RhB}

We check the resolvability of the as-synthesized AAO structure for a mixture of molecular components. Fig. 11A shows the fluorescence spectra of $\mathrm{R} 6 \mathrm{G}$ and $\mathrm{RhB}$ liquid samples with a concentration of $10^{-6} \mathrm{~mol} \mathrm{~L}^{-1}$, which were collected using a traditional fluorescence spectrometer. Fig. S7 $\uparrow$ shows the absorption and fluorescence spectra of $\mathrm{RhB}$ molecules. The result shows that under the excitation of $532 \mathrm{~nm}$ light, the fluorescence intensity of R6G is much stronger than that of RhB. Meanwhile, $2 \mu \mathrm{L}$ liquid sample was dropped on the surface of the AAO based detection chip and confocal fluorescence spectral analyses were proposed, as shown in Fig. 11B. It can be clearly found that, based on the AAO detection chip, the fluorescence intensity of $\mathrm{RhB}$ is much stronger than that of R6G molecules.

Thus, we can conclude that the AAO nano pore structure shows much better light amplification of the emission light of RhB molecules at $580 \mathrm{~nm}$ than R6G molecules at $547 \mathrm{~nm}$ under $532 \mathrm{~nm}$ light excitation. It can also been found that, under liquid conditions, the fluorescence signal of R6G molecules covered up the signal of RhB molecules. So, it is too difficult to distinguish the fluorescence signal of RhB molecules from the solution mixture of these two molecules. However, the AAO based confocal fluorescence detection chip possesses much better resolution due to its stronger light amplification of the emission light of RhB at $580 \mathrm{~nm}$. Depending on the above discussion, the detection of $\mathrm{RhB}$ molecules from the mixture sample of R6G and RhB with different volume ratios was proposed. 

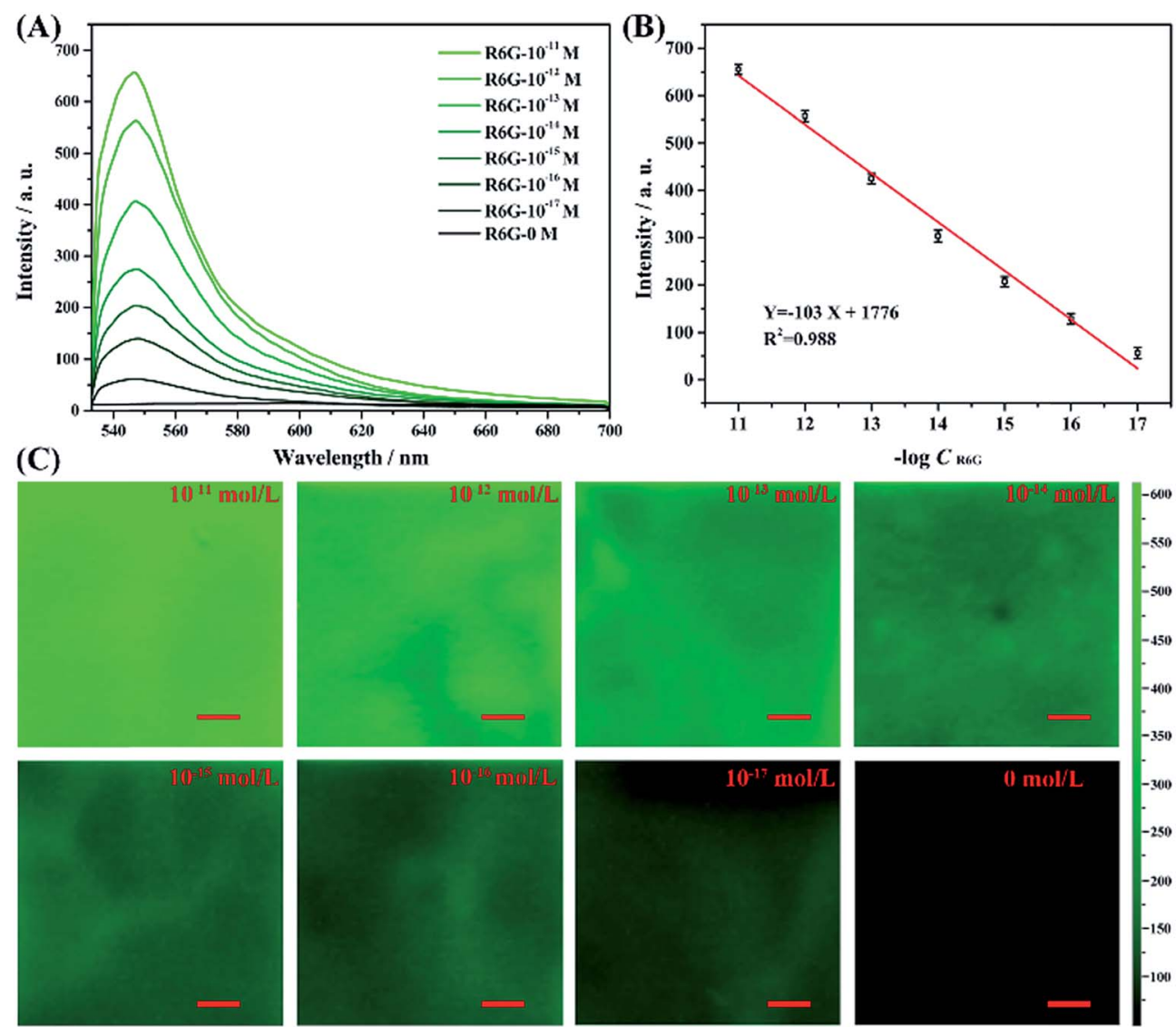

Fig. 10 The fluorescence spectra of the R6G molecules adsorbed on the AAO surface with different concentrations (A), fluorescence intensity of R6G molecules with the logarithmic value of R6G molecules (B) and the mapping result with different concentrations of R6G molecules ranging from $10^{-11} \mathrm{~mol} \mathrm{~L}^{-1}$ to $0 \mathrm{~mol} \mathrm{~L} \mathrm{~L}^{-1}(\mathrm{C})$. The scale bar indicates $30 \mu \mathrm{m}$.
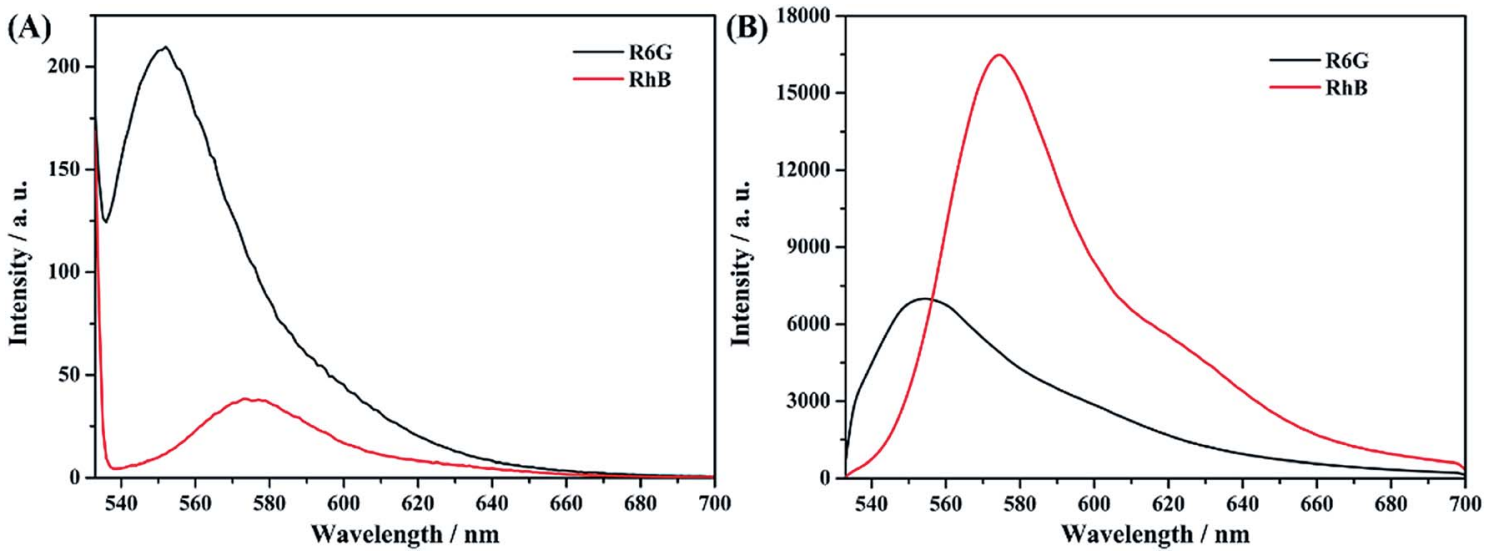

Fig. 11 Fluorescence spectra of R6G and RhB solution (A) and confocal fluorescence spectra of R6G and RhB on the AAO chip (B) with the same concentrations.

As Fig. 12A shows, the fluorescence spectra of the solution mixture shows an obvious red shift with increasing contents of $\mathrm{RhB}$ molecules. This result can be more clearly revealed by the mapping analyses with changing contents of RhB molecules (Fig. 12B). Different colors represent different fluorescence intensities of the spectra. The brightest position (white color) shifts regularly with the change in contents of the RhB molecules, which matches well with the spectral result (Fig. 12A). For comparison, the fluorescence spectra and volume-dependent mapping analysis result of the solution mixture with the same volume ratio were obtained using a traditional fluorescence spectrometer. The results are shown in Fig. S8. $\dagger$ We found from 

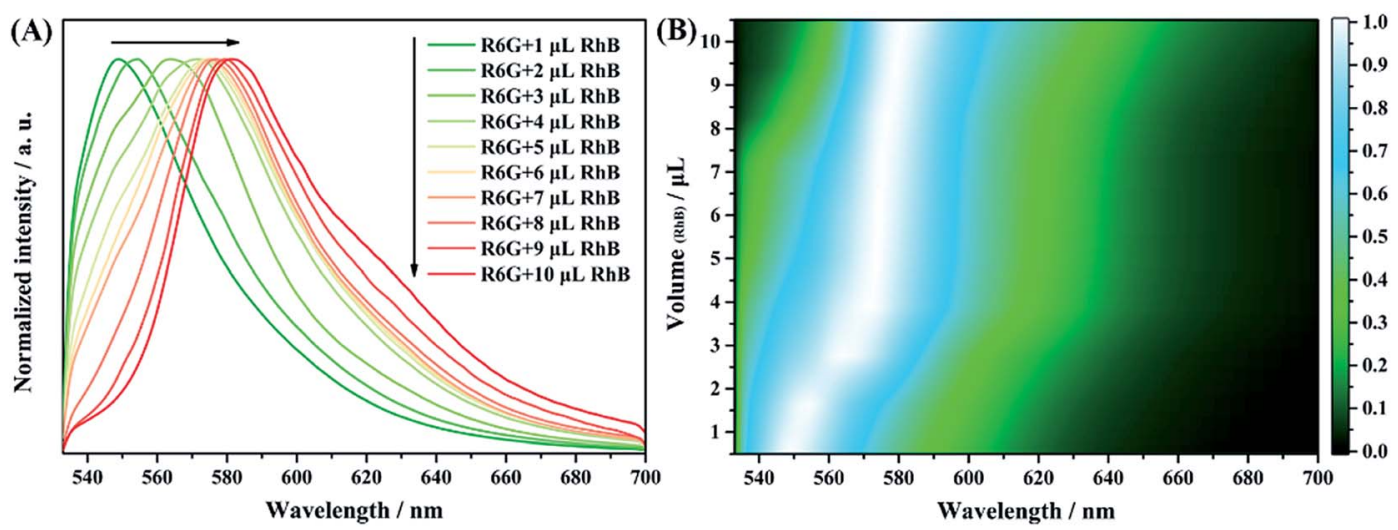

Fig. 12 Confocal fluorescence spectra of $100 \mu \mathrm{L}$ R6G mixed with different volumes of RhB from 1 to $10 \mu \mathrm{L}$ on the AAO chip (A) and fluorescence mapping analyses of the mixture components (B).

the figure that there is almost no obvious band shift with the change in contents of the RhB molecules. As is well known, the mixture of dye molecules might undergo an energy transfer process, resulting in the enhancement of the fluorescence at very low concentrations. The efficiency of the energy transfer process is strongly distance dependent. Effective FRET was observed in the case of nanocomposites using micelles, films, zeolites and nanofibers as hosts due to the restricted dimensions and spectral overlap between the donor and the acceptor. ${ }^{45-48}$ In our case, the mixed sample of R6G and RhB was deposited on the surface of the AAO template. The well-ordered nanostructures offer new opportunities for controlling the local environments of the occluded dye molecules. The nanosize pore channel of AAO can restrict the mobility of dye molecules and reduce the distance between the donor and acceptor molecules, which enhanced the energy transfer between R6G and RhB and further increased the recognition of RhB molecules among R6G molecules. Therefore, we can conclude that the AAO based detection chip shows a much better fluorescence resolution for the mixture samples under some specific detection systems, such as R6G and RhB.

\section{Conclusion}

In this paper, we combined the femtosecond laser processing technique and electrochemical anodized oxidation to fabricate array-based fluorescence LOC detection devices with each unit integrated with AAO nanoporous structures. With the assistance of femtosecond laser processing, the integration of AAO nanoporous structural units on a fixed point can be realized. This fabrication method, instead of using conventional photoresists and lithography methods, is much more simple and convenient. The as-prepared LOC devices were used for fluorescence detection analyses and the AAO nanoporous structure shows significant fluorescence enhancement properties with the fluorescence intensity of R6G molecules being enhanced 70 times over a flat surface. Furthermore, the LOC device was found to exhibit a much better spectral resolution than traditional fluorescence spectrometers in liquid samples, based on which the mixture of R6G with different contents of RhB molecules can be obviously distinguished using the fluorescence emission band shifts with changing contents of RhB molecules. Thus, the as-prepared array based LOC device is not only suitable for fluorescence enhancement analyses but also beneficial for the detection of mixture components.

\section{Conflicts of interest}

There are no conflicts to declare.

\section{Acknowledgements}

This research was supported by the National Key R\&D Program of China (2017YFB1104700), the National Natural Science Foundation (NSFC, Grant no. 61705227, 61774155, 11774340), the Jilin Provincial Science \& Technology Development Project (20180414019GH), and the Scientific research project of the Chinese Academy of Sciences (QYZDB-SSW-SYS038).

\section{References}

1 A. Manz, N. Graber and H. M. Widmer, Sens. Actuators, B, 1990, 1, 244-248.

2 F. F. Bier and S. Schumacher, Adv. Biochem. Eng./Biotechnol., 2013, 133, 1-14.

3 D. Figeys and D. Pinto, Anal. Chem., 2000, 72, 330a-335a.

4 J. Kong, L. Jiang, X. O. Su, J. H. Qin, Y. G. Du and B. C. Lin, Lab Chip, 2009, 9, 1541-1547.

5 Y. Zhang, H. Yu, J. H. Qin and B. C. Lin, Biomicrofluidics, 2009, 3, 044105.

6 C. Y. Li, X. L. Dong, J. H. Qin and B. C. Lin, Anal. Chim. Acta, 2009, 640, 93-99.

7 Y. X. Li, Y. Q. Li, B. Zheng, L. L. Qu and C. Li, Anal. Chim. Acta, 2009, 643, 100-107.

8 D. Mark, S. Haeberle, G. Roth, F. von Stetten and R. Zengerle, Chem. Soc. Rev., 2010, 39, 1153-1182.

9 H. Craighead, Nature, 2006, 442, 387-393.

10 D. G. Grier, Nature, 2003, 424, 810-816. 
11 S. K. Sia and G. M. Whitesides, Electrophoresis, 2003, 24, 3563-3576.

12 M. T. Breckenridge, T. T. Egelhoff and H. Baskaran, Biomed. Microdevices, 2010, 12, 543-553.

13 D. Irimia and M. Toner, Integr. Biol., 2009, 1, 506-512.

14 S. N. Bhatia and D. E. Ingber, Nat. Biotechnol., 2014, 32, 760772.

15 K. Sugioka and Y. Cheng, Appl. Phys. Rev., 2014, 1, 041303.

16 K. C. Phillips, H. H. Gandhi, E. Mazur and S. K. Sundaram, Adv. Opt. Photonics, 2015, 7, 684-712.

17 B. Gu, Proc. Soc. Photo-Opt. Instrum. Eng., 2004, 5339, 226230.

18 R. Buividas, M. Mikutis, T. Kudrius, A. Greicius, G. Slekys and S. Juodkazis, Lith. J. Phys., 2012, 52, 301-311.

19 N. Sudani, K. Venkatakrishnan and B. Tan, Optic Laser Eng., 2009, 47, 850-854.

20 B. N. Chichkov, C. Momma, S. Nolte, F. vonAlvensleben and A. Tunnermann, Appl. Phys. A: Mater. Sci. Process., 1996, 63, 109-115.

21 K. Sugioka and Y. Cheng, Lab Chip, 2012, 12, 3576-3589.

22 Y. L. Zhang, Q. D. Chen, H. Xia and H. B. Sun, Nano Today, 2010, 5, 435-448.

23 C. B. Schaffer, A. Brodeur and E. Mazur, Meas. Sci. Technol., 2001, 12, 1784-1794.

24 R. R. Gattass and E. Mazur, Nat. Photonics, 2008, 2, 219-225.

25 R. Osellame, G. Cerullo and R. Ramponi, Femtosecond laser micromachining: photonic and microfluidic devices in transparent materials, Springer, Berlin, 2012.

26 C. H. Lin, L. Jiang, H. Xiao, S. J. Chen and H. L. Tsai, Opt. Lett., 2010, 35, 2937-2939.

27 J. Xu, D. Wu, Y. Hanada, C. Chen, S. Z. Wu, Y. Cheng, K. Sugioka and K. Midorikawa, Lab Chip, 2013, 13, 46084616.

28 C. G. K. Malek, Anal. Bioanal. Chem., 2006, 385, 1362-1369.

29 K. Sugioka, J. Xu, D. Wu, Y. Hanada, Z. K. Wang, Y. Cheng and K. Midorikawa, Lab Chip, 2014, 14, 3447-3458.

30 A. Chen, M. M. Eberle, E. J. Lunt, S. Liu, K. Leake, M. I. Rudenko, A. R. Hawkins and H. Schmidt, Lab Chip, 2011, 11, 1502-1506.
31 X. Fang, Q. Q. Zhao, H. M. Cao, J. Liu, M. Guan and J. L. Kong, Analyst, 2015, 140, 7823-7826.

32 A. Banerjee, A. Pais, I. Papautsky and D. Klotzkin, IEEE Sens. J., 2008, 8, 621-627.

33 P. A. Quinto-Su, H. H. Lai, H. H. Yoon, C. E. Sims, N. L. Allbritton and V. Venugopalan, Lab Chip, 2008, 8, 408-414.

34 S. Kuhn, B. S. Phillips, E. J. Lunt, A. R. Hawkins and H. Schmidt, Lab Chip, 2010, 10, 189-194.

35 R. P. Jia, Y. Shen, H. Q. Luo, X. G. Chen, Z. D. Hu and D. S. Xue, Thin Solid Films, 2005, 471, 264-269.

36 R. Q. Li and H. Grebel, IEEE Sens. J., 2010, 10, 465-468.

37 M. L. Wang, G. W. Meng, Q. Huang, M. T. Li, Z. B. Li and C. L. Tang, Analyst, 2011, 136, 278-281.

38 T. Fujii, Y. Gao, R. Sharma, E. L. Hu, S. P. DenBaars and S. Nakamura, Appl. Phys. Lett., 2004, 84, 855-857.

39 T. H. Zhang, Y. He, J. J. Wei and L. Que, Biosens. Bioelectron., 2012, 38, 382-388.

40 N. Ganesh, W. Zhang, P. C. Mathias, E. Chow, J. A. N. T. Soares, V. Malyarchuk, A. D. Smith and B. T. Cunningham, Nat. Nanotechnol., 2007, 2, 515-520.

41 A. Dorfman, N. Kumar and J. I. Hahm, Langmuir, 2006, 22, 4890-4895.

42 J. W. Zhao, L. Z. Wu and J. F. Zhi, J. Mater. Chem., 2008, 18, 2459-2465.

43 C. P. Gu, J. R. Huang, N. Ni, M. Q. Li and J. H. Liu, J. Phys. D: Appl. Phys., 2008, 41, 175103.

44 H. Wang, Z. Yin, W. Xu, D. L. Zhou, S. B. Cui, X. Chen, H. N. Cui and H. W. Song, Nanoscale, 2016, 8, 10004-10009.

45 C. V. Kumar and A. Chaudhari, Microporous Mesoporous Mater., 2000, 41, 307-318.

46 B. J. Scott, M. H. Bartl, G. Wirnsberger and G. D. Stucky, J. Phys. Chem. A, 2003, 107, 5499-5502.

47 K. J. Lee, J. H. Oh, Y. Kim and J. Jang, Adv. Mater., 2006, 18, 2216-2219.

48 L. Z. Wang, Y. L. Liu, F. Chen, J. L. Zhang and M. Anpo, J. Phys. Chem. C, 2007, 111, 5541-5548. 